\title{
Finger tracking and hand recognition technologies in virtual reality maritime safety training applications
}

\author{
Evangelos Markopoulos ${ }^{1,2}$, Panagiotis Markopoulos ${ }^{3}, \mathrm{Niko} \mathrm{Laivuori}^{2}, \mathrm{Christos}_{\text {Moridis }}{ }^{4}$, Mika Luimula $^{2}$ \\ ${ }^{1}$ University College London, London UK \\ ${ }^{2}$ Turku University of Applied Sciences, Turku Finland \\ ${ }^{3}$ University of the Arts London, London UK \\ ${ }^{4}$ Manchester Metropolitan University, Manchester UK \\ e.markopoulos@ucl.ac.uk; p.markopoulos1@arts.ac.uk; niko.laivuori@edu.turkuamk.fi; \\ cmoridis@netvalue.gr; mika.luimula@turkuamk.fi
}

\begin{abstract}
The competitiveness and development of the maritime sector together with the continuous effort on increasing operations performance while reducing operations costs, drives the needs for on-board effective and qualitative training safety related issues. Virtual reality (VR) has been considered by classification societies and training organizations as a technology that can significantly improve seafarer's performance and competence with the adaptation of maritime applications developed for design simulation and gaming. This paper presents the evolution of the MarSEVR (Maritime Safety Education with VR) technology as a new concept and technology by integrating finger tracking and hand recognition technologies that increase immersiveness and user engagement within the MarISOT technology, a Green Ocean innovation composed of VR safety applications. The paper approaches this integration by addressing game design, pedagogic and cognitive neuroscience principles and challenges on the use of hand recognition and finger tracking in the MarSEVR learning episodes.
\end{abstract}

Keywords: Finger Tracking, Hand Recognition, Virtual training, Virtual Reality, Cognitive Science, Neuroscience, Maritime safety training, Shipping.

\section{INTRODUCTION}

Today's modern and technologically sophisticated ships relay significantly on the safe operations of the seafarers [1]. The maritime sector and the shipping industry in general, operates in complex environments with highly variable operational, economic, social, regulatory, political, and international challenges. This creates an operational complexity which requires from the seafarers to have the appropriate training and skills needed to manage such challenges. Maritime training and education seem to get aligned with the digital transformation powered by advanced technologies that impact the seafarer's traditional tasks [2].

As of today, training on such challenges have been approached with the use of advanced simulators for the development of seafarer competencies. Furthermore, emerging immersive technologies, such as virtual reality (VR), augmented reality (AR) and mixed reality (MR) transformed the concept and operations of maritime simulations and simulators with disruptive functionality and operations on land and at sea. However, the rapid pace of the technology evolution provocatively questions what is considered today state of the art. The integration of futuristic interactive technologies with the immersive VR and AR technologies, combined with gamification and game design principles and practices can drive the overall training experience, especially in maritime, into unexplored spaces and opportunities. Such a technological integration is demonstrated in this paper and applied on MarSEVR, an existing advanced maritime safety education technology, in an attempt to move beyond the existing blue oceans discovered with VR.

\section{THE IMMERSIVE SAFE OCEANS TECHNOLOGY}

The term Immersive Safe Ocean Technologies (ISOT) has been invented at TUAS after extensive research on developing and integrating VR and AR technologies for major maritime and shipping needs [3].

MarISOT (Maritime ISOT) emphasizes primarily on safety at sea technologies and strategies that contribute towards effective maritime safety operations. Its costeffective, portable technologies can be used on board just in time or in maritime training centers. Today MarISOT consists of four technologies namely (1) command bridge, (2) engine/machine room, (3) crane, and (4) fire safety (fig. 1). MarISOT intends to address major shipping environmental challenges such as accident prevention and sea pollution reduction.

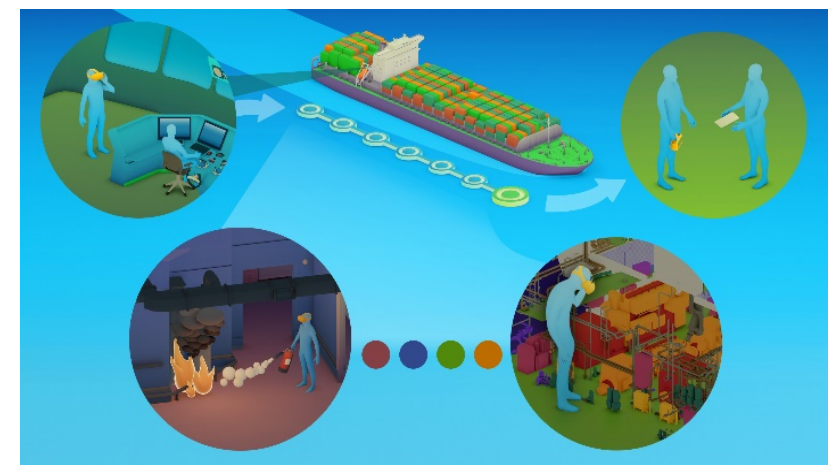

Figure 1. The MarISOT Technology

The MarISOT philosophy and its strong element of immersiveness in its VR and AR driven technologies extend business opportunities beyond Blue Ocean Strategies towards Green Ocean Strategies where operational effectiveness is driven by environmental sustainability while maintaining financial sustainability. 
Green Ocean Strategies address social, corporate, and customer needs and expectations by shifting innovation towards sustainable operations [4]. Organizations targeting Green Ocean Strategies can improve their reputation but also achieve high ESG (Environmental, Social, and Governance) ratings which are valued by their clients and investors [5]. MarISOT immersive technologies drive this transition from Blue Ocean Strategies to Green Ocean Strategies through maritime safety for clean oceans.

\section{THE MARISOT COMMAND BRIDGE APPLICATION.}

The MarSEVR (Maritime Safety Education with VR Technology) is the first MarISOT technology developed at TUAS with the support of Aboa Mare maritime specialists [6]. The technology provides practical situational awareness and decision making by replicating a ship bridge environment to be used for virtual education in maritime safety training (fig. 2).

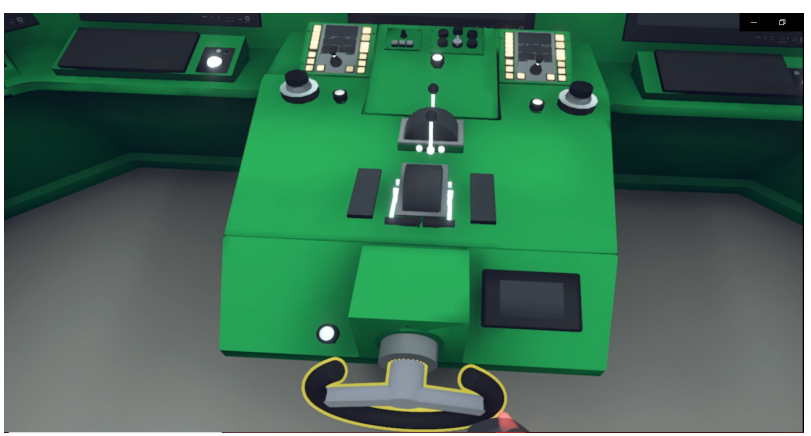

Figure 2. The MarSEVR Vessel command bridge

The vessel type used in MarSEVR is a Feeder containership 1 (1610 TEU) with the following characteristics: Length: $196.0 \mathrm{~m}$, Height of eye: $31 \mathrm{~m}$. Max speed: $20.5 \mathrm{knt}$. Displacement: 24080.0 t. Breath: $27.2 \mathrm{~m}$, Bow draft: $8.5 \mathrm{~m}$, Stern draft $9.5 \mathrm{~m}$, Type of engine: Slow Speed Diesel (1x12640kw). Type of Propeller: CPP, Thruster stern: Yes. Thruster bow: Yes

For this vessel, a training scenario has been designed on a watch change and collision avoidance situation (fig. 3).

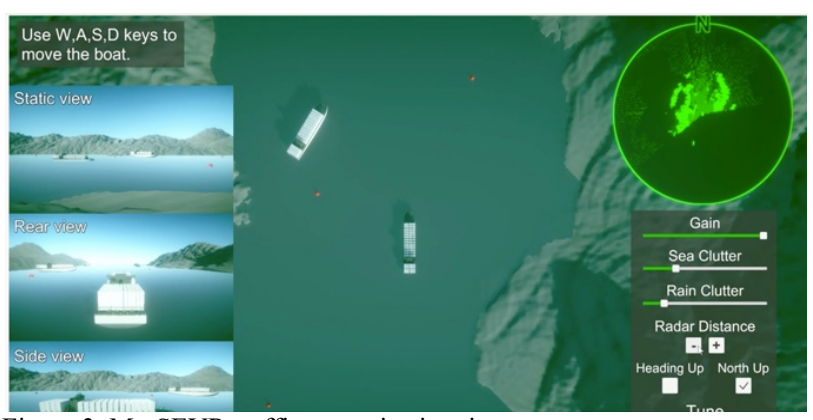

Figure 3. MarSEVR traffic scenario situation

The learning goals for the trainees are to practice their attention on the equipment's settings and status and take the actions needed to avoid a collision.

The technology has been tested in large shipping companies where experienced captains participated in the execution of this specific training scenario [7].
Despite their positive feedback on the impressive and realistic simulation of the command bridge and the scenario situation, there were concerns on the usability of the technology due to the use of hand controls (fig. 4). The users insisted that a hands-free execution can synchronize the mind with the hands and provide more accurate results by avoiding confusion and coordination mistakes.

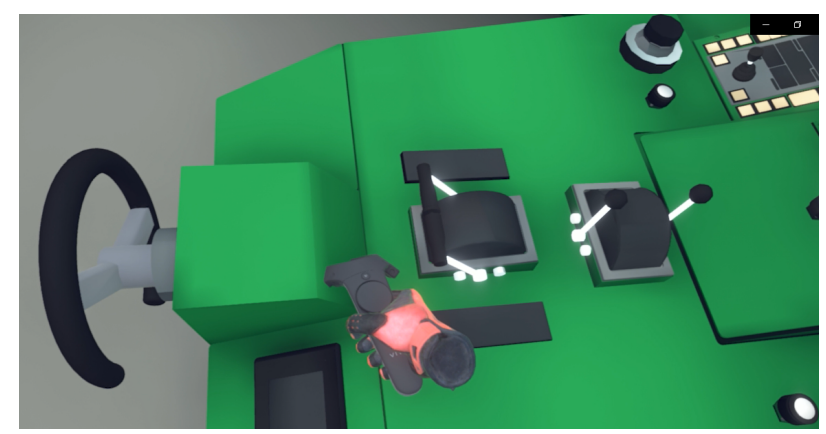

Figure 4. MarSEVR scenario execution with VR hand controls

This valuable feedback was taken into consideration and further research was conducted on intergrading finger tracking and hand recognition technologies on the MarSEVR technology. Furthermore, pedagogic and neuroscience challenges have been studied and approached with game design principles for the increase of usability and user experience in VR.

\section{VIRTUAL REALITY AND HAPTIC HUMAN COMPUTER INTERACTION ON GESTURE RECOGNITION}

The evolution of computing platforms over the last thirty years can be categorized by the eras of personal computer, mobile internet, and virtual reality based on wearable computers. In a similar way haptic human computer interaction can be categorized into the desktop haptics, the surface haptics, and the wearable haptics. VR provides todays realistic visual and auditory feedback but lacks on effective haptic feedback that can offer physical world perception through abundant haptic properties [8]. These studies are in line with the Cognitive Infocommunication principles by focusing on the challenges of making the communication of information more natural for users [9].

Haptic illusion has been widely used to meet compactsized requirement of wearable haptic devices and contribute towards a more realistic approach on the VR distinct characteristics of interaction, immersion and imagination [10].

Interaction is considered the natural interaction between the user and the virtual scene, immersion is the feeling of being part of a virtual world in the VR scene and imagination is the use multi-dimensional perception information in VR scenes to obtain the real world feelings while acquiring other feelings not available in the real world [11].

Gesture recognition has a significant role on achieving any of the VR characteristics, especially the integration of haptic technologies. Gesture is expressed consciously or unconsciously by hand, arm, or limb movement. 
Gesture recognition technology is mainly categorized into wearable sensor devices, computer vision and touch devices. Gestures interaction in VR can be categorized into wearable sensor-based devices (data gloves, inertial sensors, myoelectricity) and touch devices (touch screen, stylus pen) [10]. Finger tracking and hand recognition are two gesture interaction technologies used in the MarSEVR command bridge technology

\section{PEDAGOGIC AND COGNITIVE CHALLENGES}

A bridge simulator for maritime safety training can enforce an unnecessary cognitive load, causing cognitive overload [12] and thus significantly impair the user's learning ability. According to the cognitive load theory (one of the most important theories of learning which establishes a theoretical structure of the cognitive architecture), this is a common condition when the learner needs to integrate complex information from multiple sources and psychomotor skills. The haptic interaction and diverse visual stimuli in the context of VR simulation could enforce extra cognitive load as compared to other training modalities. For example, if users have to consider how to navigate in the virtual environment, they may be distracted from the learning task [13]. Moreover, a bridge simulator for maritime safety training often includes challenging training conditions involving high risk. Previous research findings concerning VR simulators for risk management suggest that a less constrained interface should be planned in the development of such systems to facilitate users to perceive risk accurately [14].

Unfortunately, many VR education systems suffer from complicated interaction handling of the virtual setting. This negatively affects the learner's ability to shape an elaborated mental representation of the learning task, comprehend it better, and decide how best to complete it.

Accordingly, it has been supported [15] that hand and finger tracking in the context of VR in education could decrease the learning curve when first introducing new users into virtual-reality environments. The user does not have to learn how to use the system's controllers and thus can entirely focus on the learning task. Therefore, a larger part of the user's cognitive load consists of the learning task and thus this load is beneficial to learning.

Moreover, better control of the environment can be achieved through handling down to slight movements of hands and fingers, expectantly improving muscle memory for VR manipulations/gestures and thus significantly increasing the likelihood of selecting and executing the appropriate reactions in real-world situations.

\section{GAME DESIGN CHALLENGES IN VR}

Virtual reality increased its popularity as games started to introduce technology in their attempt to become more immersive and engaging. However, the AAA style games, especially the First Person Shooter (FPS) and the RolePlaying Games (RPG) are not really VR friendly.

The main challenge for these types of games, among others, is the lack of freedom the player-character faces when moving through the world (game space/environment). This is primarily due to lack of space needed for the player to stretch out, takes steps and perform the desired body movements to enjoy the game. Another limiting factor, besides the physical space, is the sense of motion sickness players feel from the prolonged headset use. Switching the brain into another reality can have a nauseating effect if sustained for too long. To tackle these challenges, among others, VR games need to be shorter, and the gameplay mechanics must be more innovatively reworked [16].

Testing the MarISOT technology with industry experts indicated that these game design challenges exists in the professional or serious gaming applications [17]. The users indicated nausea by prolonged VR play, spatial restrictions, and the sense of limited freedom movement. Most of the seafarer officers are double the age of the average gamer, therefore the nausea challenge can be resolved with short learning episodes that can be executed in clusters towards completing a learning objective. The short episodes can also resolve the space management issue as the user will stay in the virtual space for a limited time and therefore will make less moves and steps towards completing a task.

The integration of the hand recognition and finger tracking technology provide hands free operations which increases the freedom to move and act normally. Research is also conducted on MarSERV to integrate eye tracking technologies and synchronize it with hand recognition and finger tracking in an attempt to reduce the space limitation challenge as the user will be able to spot easier objects without the need to make unnecessary moves. Effectively addressing these game design elements in professional virtual training environments can increase trainee's engagement to achieve the training goals and expectations.

\section{TECHNOLOGY IMERSIVENESS ROADMAP.}

Committed to follow the VR technology trends and challenges from the game design and the neuro cognitive science perspective, but also to satisfy the user requirements, the evolution of the MarISOT MarSEVR technology has been directed towards increasing impressiveness to obtain more effective user interfaces (UI) and immersive user experiences (UX) (fig. 5).

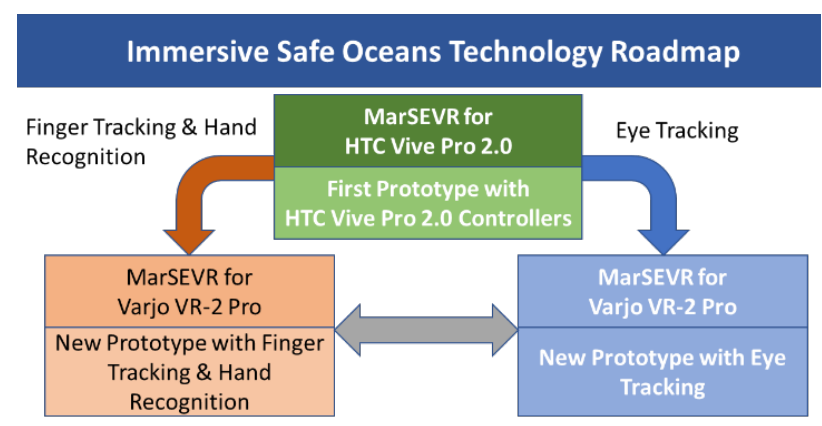

Figure 5. Technology roadmap to increase the impressiveness.

The technology was initiated with the development of the first prototype using the HTC Vive Pro 2.0 controller. Today two research initiatives run in parallel integrating through the Varjo VR-2 Pro headsets finger tracking, hand recognition and eye tracking features. In this stage and in this effort to implement hand recognition and finger tracking this research the Leap Motion Controller was used with the Varjo VR-2 Pro VR headsets. 
This light controller, of 32 grams, is an optical hand tracking module with 150x120 degrees field of view, and $80 \mathrm{~cm}$ range for user's arm length and has two infrared cameras with resolution of $640 \times 240$ pixels with 120 frames per second. [18].

The Varjo VR-2 Pro VR headsets have been integrated into the MarSEVR command bridge application using Unity game engine (version 2018.3.14f1), Ultraleap SDK for Varjo (version 4.0.0) to allow hand tracking in virtual reality, and the VR-2 Pro Unity Modules package in Unity. The original MarSEVR application has been developed with Unity in 2019 and in previous studies the HTC Vive Pro VR 2.0 headsets were used.

The Ultraleap SDK for Varjo contains tools to manage camera setup, and hand models, retrieve hand data from the sensors, and handle hand interactions with objects. In order to make objects interactable with hands, physics and specific types of interaction for each object, the related configuration is feasible. In addition, the amount of force needed to interact with buttons and toggles and events used to trigger object behavior can also be adjusted.

\section{FINGER TRACKING AND HAND \\ RECOGNITION IN A COMMAND BRIDGE VR APPLICATION.}

MarSEVR was selected as the first MarISOT application to utilize finger tracking and hand recognition due to the complexity indicated at the command bridge operation environment.

As field tests indicated that that VR inexperienced users and especially the ones without sufficient understanding of gaming and immersive technologies have difficulties to use HTC Vive Pro 2.0 controllers, the first attempt was to eliminate the use of the controllers form their hands and from their virtual sight.

This was achieved by managing to highlight a command bridge instrument once the hand was touching it. Figure 6 indicates the vessel power engine touch with the hand controls. The yellow outline on the power engine handle indicates the grip of the instrument with the hand controls.

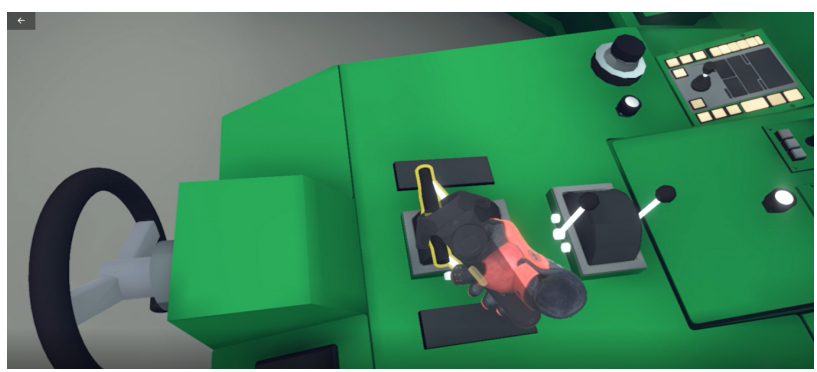

Figure 6. Vessel power engine grabbed with VR hand controls

Figure 7 indicates the same instrument touched with the hand recognition technology. The integration of Ultraleap's Motion Leap Controller offers various gestures to track and trace hand and finger movement replacing even more effectively the normal controllers. In the MarSEVR application, the users are able now to point, touch, press, grab, hold and move the command bridge instruments hands free, as they would normally do.
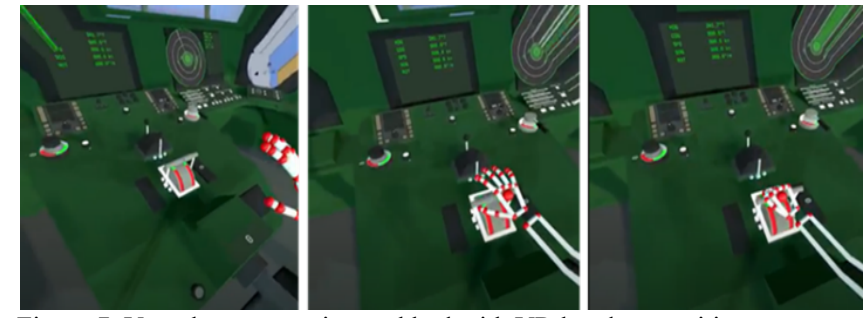

Figure 7. Vessel power engine grabbed with VR hand recognition.

Figure 8 presents a combination of such gestures on steering the ship by grabbing, holding, and moving the ship's helm (ship steering wheel).

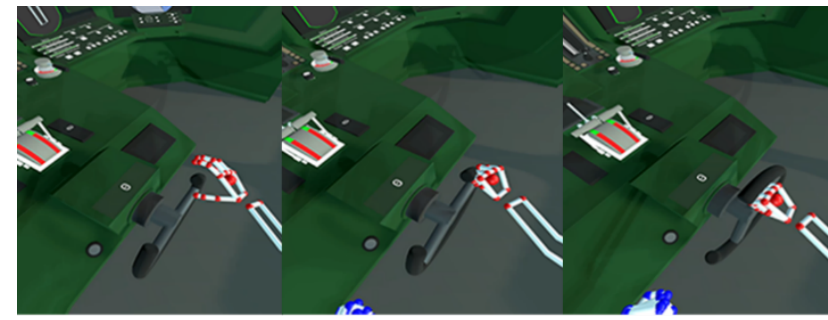

Figure 8. Grabbing, holding and moving ship's wheel based on hand recognition.

In the previous version of MarSEVR, users also found challenging to configure the ship's radar as it contains sliding bars, buttons etc. The Leap Motion Controller has intuitive gestures available and now for example the Rings button can be pointed and touched. Users are not able to feel yet the touch, but it can be visually seen, with green colour, as shown with the right hand in figure 9.

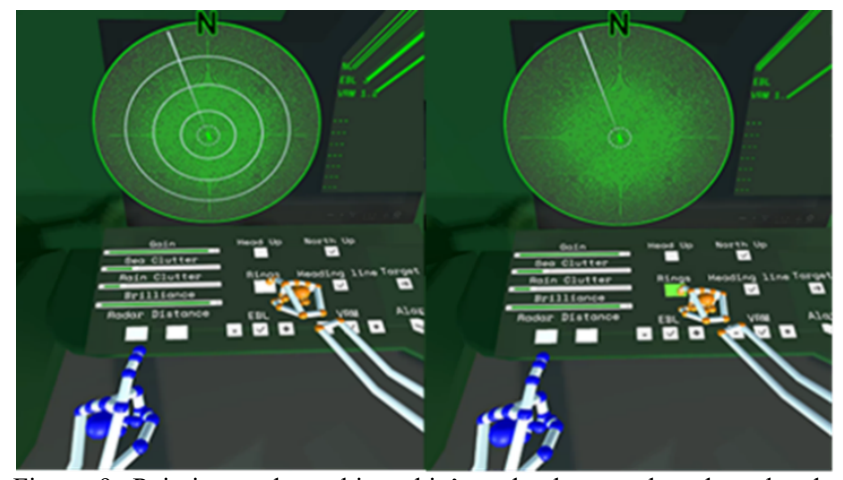

Figure 9. Pointing and touching ship's radar buttons based on hand recognition.

Another gesture, presented in figure 10, is the way users are now able to pinch objects such as the ships' manipulator. Again, when the object is touched, and prior any action on it, the object is visualized but changing its colour into green, in this case, to increase usability.

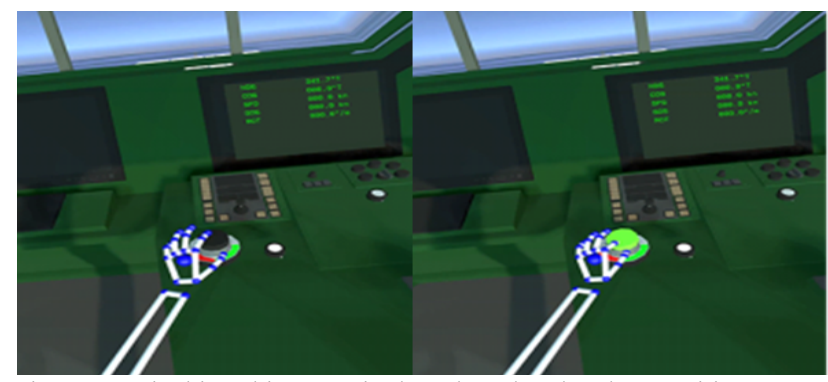

Figure 10. Pinching ship's manipulator based on hand recognition. 


\section{EXPERT EVALUATION ON THE RESULTS.}

Finger tracking offers natural movement which harmonizes the user's intent with the outcomes of the simulation. The use of VR nullifies the complication many flatscreen simulations face of offering convincing depth to objects and thus subconsciously eases the mind allowing it to focus on what it wants to do instead of how to go about doing it. The use of realistic graphics and shading complements the realistic scenarios the user is tasked with and the smart use of real-time physics in certain simulations provide a pleasant gamified experience. Other forms of gamification are seen in the clean UI that clarifies all needed information while not breaking their immersion due to their minimalistic style.

From the cognitive science perspective, both finger tracking and hand recognition offer the degree of freedom to unexperienced, with the VR technology, users. The tests conducted with experienced seafarers indicated their confusion on using the controls properly to navigate in the virtual space. This impacted their response time, decision making, thinking process, attention, and concentration, that creates unnecessary frustration and psychological disturbance from errors made due to lack of moving and acting freely. Both technologies contribute to the concentration of the user on the learning tasks and objectives without stress and anxiety. They align the mind with the body to delivering what the users wants to do and what they wanted to do.

\section{COGNITIVE AND NEURO SCIENCE PERSPECTIVE}

Previous studies have stressed the prospective of highresolution encephalograph (EEG) to be used with VR systems [19], empowering effective representation of bodily stimuli. The alpha band $(8-12 \mathrm{~Hz})$ brain oscillation over central brain areas (mu rhythm) has been related to sensorimotor processing [18]. Tasks related to action, for example, action execution and observation [19], as well as motion perception [20] have been made known to suppress the mu rhythm over those areas of the brain. Importantly, in the context of VR, alpha band in the medial prefrontal cortex (mPFC) has been correlated with the degree of experimentally manipulated self-location [17], where participants experienced their self to be localized in the VR environment.

By merging VR with cognitive neuroscience and employing high-resolution EEG, there is a great potential of getting valuable insights into the brain mechanisms of human bodily self-consciousness. This will not only help improve neuroscience knowledge, but will also provide a solid ground for adapting VR systems to human cognitive processing.

Furthermore, the use of EEG provides a direct interface to the brain which combined with VR can be a huge step forward [20]. Several studies [21, 22, 23] have shown that EEG signals can be used to transfer human intentions to computers using Brain-Computer Interface (BCI), thus enabling user interaction through the human mind. Thus, in the near future BCI integrated into VR environments could provide a new generation of more advanced VR systems.

\section{GAME DESIGN LEARNING EFFECT ON FINGER TRACKING AND HAND RECOGNITION}

Merging Virtual Reality with game-based approaches enhances training and learnings methods and practices. Such realistic learning environments transform the trainees from passive observers into active participants, engaging them in the development of exploration-based learning paradigms [24]. This promising future in professional education is supported with the wide availability of affordable software and hardware technologies for VRenvironments. Likewise, software technologies such as Unreal Engine and Unity, used mainly for games development, support fast development of user interactions with the VR-environment.

MarISOT followed this recipe by using Unity for the software development and Varjo-V2 together with Ultraleap's Motion Leap Controller for the VR experiences and the hand recognition functionality, in its attempt to achieve the high levels of interactivity serious games offer.

Serious games facilitate active and critical learning by providing a learner-cantered approach totally different for the teacher-centric found in traditional teaching environments. The trainee takes control of the learning process and feels the interactive learning [25].

Furthermore, this control becomes stronger when the trainee performs hands free activities as in a natural environment. The recognition of hand gestures is a challenging task for the high degrees of freedom on hand motion. This was also a user requirement of the pervious MarSEVR version towards achieving the freedom needed to perform critical operations. Hand tracking allows the trainees to be more expressive and connect on a deeper level with the technology. Trainees have the freedom to use their own hands in VR without controllers or other peripheral devices and enjoy the training without hesitation or delays on performing an action. The finger tracking and hand recognition technology turns virtual education into a game, a serious game, as it is intended to be.

\section{MARITIME INDUSTRY NEEDS AND TRENDS}

The maritime sector can be characterized by its high turnover on seafarers [26] and the demanding training regulations needed for a ship to sail safely [27]. Whether onboard or onshore, the knowledge and skills required to work in the maritime sector increases the need for specialized skills. Today there are more than 1.6 million seafarers worldwide and the demand for highly skilled seafarers and officers is growing [28] together with the need for effective and efficient but also affordable education and training.

This reality shifts maritime educations training from the traditional and costly training methods to more effective, engaging and immersive digital environments. Virtual Reality, Augmented Reality and Mixed Reality with emerging head mounted display (HDM) technologies create a market for new products and services that can be offered to the maritime industry. The high-cost simulators (full mission bridge or engine room facilities) which seem to have high profit for the service providers but also high cost for the shipping companies, have also high installation, installation, maintenance, and upgrading costs [29]. 
On the other hand, the decreasing price per unit on personal HMD hardware and software increases the volume of individual users in the maritime industry and offers immersive, reliable and accurate training. The enhanced MarISOT with hand recognition and finger tracking technologies can provide immersive and realistic maritime training of all types (team training, operator training, decision making training, procedure training, and maintenance training [30]) and under all performance capabilities and functionalities training classes (fullmission, multitask, limited task, and special tasks [31])

\section{IMPACT TO THE MARITIME SECTOR}

$\mathrm{VR}, \mathrm{AR}$ and gamification technologies drive maritime education trends into new dimensions with direct impact on the industry operations. Furthermore, the integration of technologies such as hand recognition and finer tracking give a futuristic impression that can pass the wrong message. Maritime experts believe that such technologies introduced into maritime training need to promote more the learning outcomes and motives than the technologies themselves. Failing to address specifically the impact and value of such technologies in maritime educations results into a major buzz in the world of maritime training, with many to be concerned that such solutions are kind of expensive gimmicks [32]. This can be true, but such costs can be absorbed, and leverage, the state-of-the-art investments continuously made in the shipping industry.

Mega ships, smart ships, and soon autonomous ships drive the maritime sector and its demand for better educated seafarers [33]. VR and AR indicate working performance improvements with significant results in the manufacturing sector where skilled expertise is required. VR and AR can also help more workers to do high-skill jobs, improve their performance, and grow the industrial productivity that can ultimately be translated into higher wages [34]. The shipping industry indicates serious employee retention issues mostly related with the employee and employer satisfaction on the job, the good working conditions, and the opportunities for career progression [35]. MarISOT technologies impact employee productivity and career development, protecting at the same time the investments made in the shipping industry on modern vessel acquisition or vessel technological upgrades.

\section{AREAS OF FURTHER RESEARCH}

The finger tracking and hand recognition technologies integrated into the MarISOT's MarSEVR technology will soon get integrated with eye tracking technologies. This research runs in parallel with the Varjo VR-2, the most advanced professional VR headset. Varjo-VR2 allows users to look around within photo-realistic environments, but also get a closer look at an object by simply leaning closer to it as they would do in real life [36].

These new technological usability functionalities will be extended to the other technologies of MarISOT with ShipSEVR to be the next. ShipSEVR, developed with the contribution of Wärtsilä, projects at the trainee's eyes a portable digital technical library, accessibility to engine and enginee room simulations, schematics, documentation, and technical diagrams [37]. Hands free operations with hand recognition and finger tracking together with eye tracking through the Varjo V2 technology will provide easiness to access, read, and analyze schematics details, search, store technical documentation and optimize the ship engine room operations and engine maintenance.

\section{CONCLUSIONS}

This paper demonstrated the integration of the finger tracking and hand recognition technologies of VR in the MarISOT technology. This integrations weas approached from a multidisciplinary perspective. The cognitive neuroscience dimension indicated the pedagogical and behavioral impact of such technologies on the trainee. This can be supported furthermore with behavioral biometrics since every person has a unique way to be expressed with individual behavioral characteristics (body movement, coordination, usage, etc) [38]. To increase the engagement of the trainee, challenges in game design and user experience in VR have been studied. The maritime sector and the shipping industry rely significantly on the updated training and readiness of the seafarers. MarISOT can provide a low-cost on-board environment that can contribute to sea safety while maintaining environmental and sustainable vessel operations. The evolution of MarISOT has been shifted from an on-board VR training Blue Ocean technology into a sea safety sustainable Green Ocean technology.

\section{ACKNOWLEDGEMENT}

The authors would like to thank everyone who have participated in the development of this training VR technology. This work was supported by Business Finland and Turku University of Applied Sciences.

\section{REFERENCES}

[1] Baldauf, M.; Dalaklis, D.; Kataria, A. Team training in safety and security via simulation: A practical dimension of maritime education and training. In Proceedings of the International Technology, Education and Development Conference, Valencia, Spain, 7-8 March 2016. 2.

[2] Mallam, S. Distributed Participatory Design in Multidisciplinary Engineering Projects: Investigating a Sustainable Approach for Ship Design \& Construction; Chalmers University of Technology: Gothenburg, Sweden, 2016.

[3] Markopoulos, E. and Luimula, M. Immersive Safe Oceans Technology: Developing Virtual Onboard Training Episodes for Maritime Safety. Future Internet 2020, 12, 80.

[4] Markopoulos E., Kirane I.S., Piper, C. and Vanharanta, H. Green ocean strategy: Democratizing business knowledge for sustainable growth. In Advances in Intelligent Systems and Computing; Springer Science and Business Media: Berlin, Germany, 2019; Vol. 1026, pp. 115-125.

[5] Markopoulos, E., Kirane, I.S., Gann, E.L., and Vanharanta, H. A democratic, green ocean management framework for Environmental, Social and Governance (ESG) compliance. In Advances in Intelligent Systems and Computing; Springer Science and Business Media: Berlin, Germany, 2020; Vol 1152, pp. 21-33.

[6] Markopoulos, E., Lauronen, J., Luimula, M., Lehto, P. and Laukkanen, S. Maritime safety education with VR technology (MarSEVR). In Proceedings of the 9th IEEE Conference on Cognitive Infocommunications, Naples, Italy, 23-25 October 2019; pp. 283-288.

[7] Virtual Reality Finnish Disruptive Innovation transforms Maritime Safety Training and Education. 30 October, 2019. URL: https://www.allaboutshipping.co.uk/2019/10/30/191293/

[8] Wang D., Guo Y., Liu S., Zhang Y., Xu W., and Xiao J. Haptic display for virtual reality: progress and challenges. Virtual Reality \& Intelligent Hardware. 2019, 1 (2) : 136-162 
[9] Baranyi P, Csapo A, "Definition and synergies of cognitive infocommunications," Acta Polytechnica Hungarica, vol. 9(1), pp. $67-83,2012$.

[10] Huang H-M., Rauch U., and Liaw S-S. Investigating learners' attitudes toward virtual reality learning environments: Based on a constructivist approach. Computers \& Education. Volume 55, Issue 3 , November 2010, Pages 1171-1182

[11] Li Y., Huang J., Tan F., Wang H., Dai G. I. Gesture interaction in virtual reality. Vutua1 Reality \& Intelligent Hardware,2019,1(1):84-112

[12] J. Sweller J., Cognitive load during problem solving: effects on learning. Cogn Sci, 12 (2) (1988), pp. 257-285

[13] Bowman, D. A., Koller, D., and Hodges, L. F. Travel in immersive virtual environments: An evaluation of viewpoint motion control techniques. In Proceedings of the Virtual Reality Annual International Symposium, 45-52, 1997

[14] Vitor A. M. Jorge, Wilson J. Sarmiento, Anderson Maciel, Luciana Nedel, César A. Collazos, Frederico Faria, and Jackson Oliveira. 2013. Interacting with danger in an immersive environment: issues on cognitive load and risk perception. In Proceedings of the 19th ACM Symposium on Virtual Reality Software and Technology (VRST'13). ACM, New York, NY, USA, 83-92.

[15] Dean D., Millward J., Mulligan L., Saleh I., Wise C. and Higgins G., Evaluating Alternative Input Techniques for Building and Construction VR Training, 2018 IEEE International Conference on Teaching, Assessment, and Learning for Engineering (TALE), Wollongong, NSW, 2018, pp. 1001-1004

[16] Will VR Change Game Design in The Future? May 12, 2018 URL: https://www.gamedesigning.org/gaming/vr-change-game-design/

[17] Lauronen J., Ravyse W., Salokorpi M., Luimula M. (2020) Validation of Virtual Command Bridge Training Environment Comparing the VR-Training with Ship Bridge Simulation. In: Stanton N. (eds) Advances in Human Aspects of Transportation. AHFE 2020. Advances in Intelligent Systems and Computing, vol 1212. Springer. pp.444-451

[18] What are the specifications of the Leap Motion Controller? December 23, 2019. URL: https://support.leapmotion.com/hc/enus/articles/360004476658-What-are-the-specifications-of-theLeap-Motion-Controller-)

[19] B. Lenggenhager, P. Halje and O. Blanke, "Alpha band oscillations correlate with illusory self-location induced by virtual reality", European Journal of Neuroscience, vol. 33, no. 10, pp. 1935-1943, 2011. Available: 10.1111/j.1460-9568.2011.07647.x.

[20] J. Pineda, "The functional significance of mu rhythms: Translating "seeing" and "hearing" into "doing", Brain Research Reviews, vol. 50, no. 1, pp. 57-68, 2005. Available: 10.1016/j.brainresrev.2005.04.005.

[19] H. Gastaut, "Electrocorticographic study of the reactivity of rolandic rhythm", Rev. Neurol., vol. 87, pp. 176 - 182, 1952.

[20] E. ULLOA and J. PINEDA, "Recognition of point-light biological motion: $\mathrm{Mu}$ rhythms and mirror neuron activity", Behavioural Brain Research, vol. 183, no. 2, pp. 188-194, 2007. Available: 10.1016/j.bbr.2007.06.007.

[21] Y. Zhang, Z. Zhu, and Z. Yun. Empower VR art and AR book with spatial interaction. In Int'l Symp. on Mixed and Augmented Reality, pp. 274-279. IEEE, 2016

[22] W. Neto, K. Shimizu, H. Mori, and T. Rutkowski. Virtual reality feedback environment for brain computer interface paradigm using tactile and bone-conduction auditory modality paradigms. In $15^{\text {th }}$ Int'l Symp. on SCIS, pp. 469-472. IEEE, 2014.

[23] J. Soeiro, A. Cl'audio, M. Carmo, H. Ferreira. Mobile solution for brain visualization using augmented and virtual reality. In 20th Int'l Conf. on Information Visualisation, pp. 124-129. IEEE, 2016.

[24] Checa, D., Bustillo, A. A review of immersive virtual reality serious games to enhance learning and training. Multimed Tools Appl 79, 5501-5527 (2020).

[25] Stapleton, A. (2004) Serious Games: Serious Opportunities. Paper presented at the Australian Game Developers' Conference, Academic Summit, Melbourne, VIC

[26] Bhattacharya Y.: Employee Engagement as a Predictor of Seafarer Retention: A Study among Indian Officers. The Asian Journal of Shipping and Logistics. Volume. 31, Issue 2, pp. 295--318 (2015)
[27] European Commision. Refitting the EU legislation on seafarers' training and certification. Commission Staff Working Document. Brussels, 24.5.2018

[28] Global Supply and Demand for Seafarers. Available online: http://www.ics-shipping.org/shipping-facts/ shipping-and-worldtrade/global-supply-and-demand-for-seafarers (accessed on 25 November 2019).

[29] Baldauf, M.; Dalaklis, D.; Kataria, A. Team training in safety and security via simulation: A practical dimension of maritime education and training. In Proceedings of the International Technology, Education and Development Conference, Valencia, Spain, 7-8 March 2016.

[30] Castells, M.L.; Ordás, S.; Barahona, C.; Moncunill, J.; Muyskens, C.; Hofman, W.; Cross, S.; Kondratiev, A.; Boran-Keshishyan, A.; Popov, A.; et al. Model course to revalidate deck officers' competences using simulators. Wmu J. Marit. Aff. 2015, 15, 163185

[31] Drown, D.F.; Lowry, I. A Categorisation and Evaluation System for Computer Based Ship Operation Training Simulators. Natl. Acad. Sci. Eng. Med. 1993, 1, 103-114.

[32] VR in maritime training: focus on what motivates learning not the technology. Nov 22, 2019. URL: https://www.seatrademaritime.com/technology/vr-maritime-training-focus-whatmotivates-learning-not-technology

[33] From Seaman to SPACEman: A Look at Virtual Reality \& Augmented Reality for Seafarers. 03 Apr 2017. URL: https://informaconnect.com/virtual-reality-augmented-reality-forseafarers/

[34] Augmented Reality Is Already Improving Worker Performance. Harvard Business Review. March 13, 2017. URL https://hbr.org/2017/03/augmented-reality-is-already-improvingworker-

performance?utm campaign $=$ hbr\&utm source=twitter\&utm medi $\underline{\text { um}}=$ social

[35] Pan X. and Hamilton A. Why and how to use virtual reality to study human social interaction: The challenges of exploring a new research landscape. British Journal of Psychology, 109, pp. 395-417 (2018)

[36] Varjo VR-2 virtual reality headset delivers "human-eye resolution". 30 November 2019. URL: https://newatlas.com/vr/varjo-vr-2virtual-reality-headset/

[37] Markopoulos, E., Luimula, M., Porramo, P., Pisirici, T., and Kirjonen. A. Virtual Reality (VR) safety education for ship engine training on maintenance and safety (ShipSEVR). In Proceedings of the 11th International Conference on Applied Human Factors and Ergonomics (AHFE), San Diego, USA, 16-20 July 2020.

[38] Pfeuffer K. J. Geiger M.J., Prange S., Mecke L., Buschek D.: Behavioral Biometrics in VR: Identifying People from Body Motion and Relations in Virtual Reality. In: Proceedings of the CHI '19: CHI Conference on Human Factors in Computing Systems. May 2019 Paper No. 110. Pp. 1--12 (2019) 